\title{
Dynamical model selection near the quantum-classical boundary
}

\author{
Jason F. Ralph, ${ }^{1, *}$ Marko Toroš,${ }^{2, \dagger}$ Simon Maskell,,${ }^{1, \dagger}$ Kurt Jacobs, $, 3,4,5, \S$ Muddassar Rashid, ${ }^{2}$ \\ Ashley J. Setter, ${ }^{2}$ and Hendrik Ulbricht ${ }^{2}$ \\ ${ }^{1}$ Department of Electrical Engineering and Electronics, University of Liverpool, Brownlow Hill, Liverpool L69 3GJ, United Kingdom \\ ${ }^{2}$ Department of Physics and Astronomy, University of Southampton, University Road, Southampton SO17 1BJ, United Kingdom \\ ${ }^{3}$ U.S. Army Research Laboratory, Computational and Information Sciences Directorate, Adelphi, Maryland 20783, USA \\ ${ }^{4}$ Department of Physics, University of Massachusetts at Boston, Boston, Massachusetts 02125, USA \\ ${ }^{5}$ Hearne Institute for Theoretical Physics, Louisiana State University, Baton Rouge, Louisiana 70803, USA
}

(Received 28 November 2017; revised manuscript received 2 May 2018; published 6 July 2018)

\begin{abstract}
We discuss a general method of model selection from experimentally recorded time-trace data. This method can be used to distinguish between quantum and classical dynamical models. It can be used in postselection as well as for real-time analysis, and offers an alternative to statistical tests based on state-reconstruction methods. We examine the conditions that optimize quantum hypothesis testing, maximizing one's ability to discriminate between classical and quantum models. We set upper limits on the temperature and lower limits on the measurement efficiencies required to explore these differences, using an experiment in levitated optomechanical systems as an example.
\end{abstract}

DOI: 10.1103/PhysRevA.98.010102

Introduction. There are a number of ways in which a system can be determined to be quantum mechanical. Typically, the system must be isolated from extraneous noise and operated at very low temperatures, so that the system is in a ground state or another low-lying energy state. The system can be subjected to a series of individual or joint measurements to build up a picture of the state (as in interference experiments and state tomography [1-6]) or manipulated using an external field to demonstrate superposition states (such as avoided crossings in the observed energy spectra [7-10]). These experiments provide direct evidence of quantum behavior but they can be difficult to perform when the system has several degrees of freedom and large numbers of measurements are required.

More efficient alternatives have been devised with the growth of quantum information as a subject area. Specific sequences of measurements can be applied to ascertain whether the system contains nonclassical correlations (entanglement) associated with quantum behavior [11-13]. Entanglement witnesses do not necessarily allow an experimentalist to quantify the degree of entanglement, but they do allow one to say that entanglement is present and, hence, that the system is quantum mechanical rather than classical. All of these methods are intended to provide direct evidence that the system is manifestly nonclassical, e.g., discrete energy levels, interference, superposition states, and entanglement.

An alternative approach is to try to determine whether the system dynamics are quantum rather than classical. An elegant approach to this task is to use the technique of quantum hypothesis testing [14,15]. In situations where direct experiments are

\footnotetext{
*jfralph@liverpool.ac.uk

${ }^{\dagger}$ m.toros@soton.ac.uk

${ }^{\ddagger}$ smaskell@liverpool.ac.uk

§kurt.jacobs@umb.edu
}

not possible, or are beyond the reach of current experiments, this method can also be used to inform future work, explore regions of parameter space, and to focus experimental efforts. This is the motivation for the current Rapid Communication.

In this Rapid Communication, we use the quantum hypothesis testing approach, often referred to as model selection in classical Bayesian inference [16], to construct a general method of model selection, which is an alternative to state-reconstruction-based statistical tests. We reformulate the problem as a Neyman-Pearson decision rule and quantify the accuracy of the selected model using the confusion matrix. As an example application, we devise an experiment for optically levitated systems [17-20], and we optimize the Hamiltonian parameters to enhance the distinguishability of quantum and classical dynamics. The proposed experiment does not require complicated preparation and measurement protocols, but relies only on the detected photocurrent [21]. Quantum behavior has not yet been established with such massive systems, and improving the understanding of where and when such evidence might be available is an important open question. We demonstrate that two experimental parameters, the effective temperature and the efficiency of the continuous measurement, are critical to the ability to distinguish between quantum and classical stochastic dynamics in this system.

Dynamical models. A model is composed of three elements: (i) the description of the system, i.e., the state, (ii) the dynamical law, and (iii) the detection process. In this Rapid Communication, we consider nonrelativistic, single-particle, classical and quantum dynamics with a diffusive, Markovian environment, subject to continuous monitoring of the position of the particle. We denote the state by $S_{c}$, the measured time trace by $I_{\text {exp }}$, and the measured position by $\tilde{q}$ (either classical or quantum). Note, however, that these assumptions are not essential, but only a matter of convenience of presentation, and could, at least in principle, all be relaxed. In particular, 
TABLE I. Quantum and classical dynamical models given by Eqs. (1) and (2). $P_{c}$ and $\hat{\rho}_{c}$ denote the conditional probability density and conditional statistical operator, respectively. $q$ and $p$ are the classical phase-space variables, i.e., position and momentum, respectively, $q(\hat{q})$ denotes the classical (quantum) position observable, $H(\hat{H})$ denotes the classical (quantum) Hamiltonian observable, and $t$ is the time variable. $\mathbb{E}_{t}[\cdot]$ denotes the expectation value with respect to the conditional state $S_{c}(t)$ at time $t$.

\begin{tabular}{lcc}
\hline \hline Symbol & Classical & Quantum \\
\hline$S_{c}$ & $P_{c}(q, p ; t)$ & $\hat{\rho}_{c}(t)$ \\
$\mathcal{K} \cdot$ & $\{H, \cdot\}_{\mathrm{Pb}}$ & $-\frac{i}{\hbar}[\hat{H}, \cdot]$ \\
$\mathcal{D}[\tilde{q}] \cdot$ & $-\frac{\hbar^{2}}{8} \frac{\partial^{2}}{\partial p^{2}}$. & $\frac{1}{8}[\hat{q},[\hat{q}, \cdot]]$ \\
$\mathcal{H}[\tilde{q}] \cdot$ & $q-\mathbb{E}_{t}[q]$ & $\left(\hat{q}-\mathbb{E}_{t}[\hat{q}]\right) \cdot+$ H.c. \\
$\mathbb{E}_{t}[\cdot]$ & $\iint d q d p \cdot P_{c}(q, p ; t)$ & $\operatorname{tr}\left[\cdot \hat{\rho}_{c}(t)\right]$ \\
\hline \hline
\end{tabular}

the analysis for general, nonrelativistic, diffusive, Markovian models can be carried out in full analogy with the analysis presented in this Rapid Communication (see Appendix A).

Under these assumptions, the state $S_{c}$ formally evolves according to [22-24] (in Itô form):

$$
d S_{c}=\mathcal{K} S_{c} d t+\gamma \mathcal{D}\left[\frac{\tilde{q}}{\sigma}\right] S_{c} d t+\sqrt{\eta \gamma} \mathcal{H}\left[\frac{\tilde{q}}{\sigma}\right] S_{c} d W
$$

where $\mathcal{K} \cdot, \mathcal{D}[\tilde{q}] \cdot$, and $\mathcal{H}[\tilde{q}] \cdot$ denote the Hamiltonian, diffusive, and detection terms [25], respectively; $W$ is a zero mean Wiener process; $\sigma, \gamma$ denote a characteristic length scale and frequency of the experiment, respectively; and $\eta$ is the efficiency of the measurement, which is defined to be the ratio between the power due to the recorded measurement signal relative to other sources of noise. Inefficient measurements may arise from loss of signal or corruption of the signal by additional, unprobed environmental degrees of freedom. The detected signal $I_{\text {exp }}(t)$ during an interval, $t \rightarrow t+d t$, is related to the Wiener process by

$$
I_{\exp }(t+d t)-I_{\exp }(t)=d I_{\exp (t)}=\sqrt{\eta} \mathbb{E}[q] d t+\frac{d W}{\sqrt{\gamma}},
$$

where $\mathbb{E}[\cdot]$ denotes the expectation value with respect to the state $S_{c}$.

For a given experimental signal, $I_{\exp }(t)$, the conditional evolution of the state can be found by inverting Eq. (2) to find a series of stochastic increments, $d \tilde{W}\left(t \mid d I_{\exp (t)}\right)$, to insert back into Eq. (1). The resultant conditional state, $S_{c}$, describes the knowledge about the state of the system, as derived from the measurement record. In classical state estimation, the stochastic increments are often called the innovation terms [26] because they represent the difference between the actual measurement taken and the measurement expected from the conditioned state during each time increment.

The explicit expression for the terms in Eqs. (1) and (2) are given in Table I. The quantum and the corresponding classical models are related by the following formal prescription: replace the quantum observables $\hat{O}$ with the corresponding classical observables $O$, and commutators with Poisson brackets, i.e., $[\cdot, \cdot] \rightarrow i \hbar\{\cdot, \cdot\}_{\mathrm{Pb}}$. Note, however, that as far as the model selection is concerned, the classical and quantum models could be very different or even completely unrelated.
Decision rule. We now consider a collection $M$ of dynamical models, which we denote by $m_{k} \in M(k=1, \ldots, N)$ : these can be either quantum or classical [see Eqs. (1) and (2) and Table I]. Before data collection, we suppose that each model is equally likely, which mathematically translates to setting the a priori probabilities to be equal, i.e., the initial probability of model $m_{j}$ is $p_{0}\left(m_{j}\right)=\frac{1}{M}$. After data collection, the goal is to select the model $m_{j} \in M$ that gives the best description of the collected data, i.e., that fits best the recorded time-trace signal $I_{\text {exp }}$.

According to the Bayes decision rule, a model $m_{j}$ is the best considered model given the detected signal $I_{\text {exp. }}$, if $\forall k \neq j$ we have $p\left(m_{j} \mid I_{\text {exp }}\right)>p\left(m_{k} \mid I_{\text {exp }}\right)$. However, in some situations, the data $I_{\exp }$ is insufficient to select a given model with certainty, e.g., two models might have experimental predictions that are not distinguishable. It is then useful to introduce an acceptance region $A=\left\{I \mid 1-\max \left[p\left(m_{k} \mid I\right)\right]>\tau\right\}$, where $I$ denotes all possible signals, $\tau$ is a threshold parameter, and max denotes the maximization over $m_{k} \in M$. In the case $I_{\text {exp }} \in A$, we apply Bayes decision rule for minimum error, otherwise we conclude the data is inconclusive, i.e., $I_{\exp }$ is in the so-called rejection region.

The Bayes decision rule and the acceptance region form a two-stage selection: we can combine these two stages by considering an alternative decision rule. Specifically, we consider the Neyman-Pearson decision rule, which has a builtin acceptance threshold parameter $\mu$ for the likelihood ratio $[14,15]$. Specifically, one selects model $m_{j}$, given the detected signal $I_{\text {exp. }}$, if $\forall k \neq j$ we have

$$
\frac{p\left(m_{j} \mid I_{\text {exp }}\right)}{p\left(m_{k} \mid I_{\text {exp }}\right)}>\mu .
$$

Note that the two decision rules coincide for $\mu=1, \tau=1$, and under the assumption of equal a priori probabilities. In the rest of this Rapid Communication, we will use the latter, more compact rule, given by Eq. (3).

To apply the decision rule, we are left to specify how to obtain $p\left(m_{k} \mid I_{\text {exp }}\right)$. Without loss of generality, we assume that the time trace is given from $t=0$ to $t=t^{\prime}$, and that the detector has a finite integration time $\Delta t$, such that $n \Delta t=t^{\prime}$. We now use the property of pairwise independence of the detected signals in each interval $\Delta t$ to obtain an update equation for each of the model probabilities [16]:

$$
p\left(m_{k} \mid I_{\exp , 0: t^{\prime}}\right) \propto p_{0}\left(m_{k}\right) \prod_{t=\Delta t: t^{\prime}} p\left(\Delta I_{\exp , t} \mid m_{k}, I_{\exp , 0: t-\Delta t}\right),
$$

where $I_{\exp , 0: t^{\prime}}$ on the left-hand side denotes the total time trace from time $t=0$ to $t=t^{\prime}, p_{0}\left(m_{k}\right)$ is the initial probability assigned to the model $m_{k}$, and $\Delta I_{\exp , t}$ on the right-hand side is the signal in the interval $[t, t-\Delta t]$. The probability updates are generated using

$$
p\left(\Delta I_{\exp , t} \mid m_{k}, I_{\exp , 0: t-\Delta t}\right)=\frac{1}{\sqrt{2 \pi \Delta t}} \exp \left(-\frac{\left(\Delta W_{t}^{\left(m_{k}\right)}\right)^{2}}{2 \Delta t}\right),
$$

where the increments (innovations) are given by

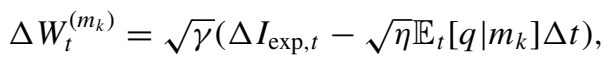


and where $\mathbb{E}_{t}\left[q \mid m_{k}\right]$ is the expected value of $q$, given the dynamical model $m_{k}$ and the associated conditional state. The probabilities for each of the possible models are updated after each time step using Eq. (4) and then normalized such that $\sum_{k} p\left(m_{k} \mid I_{\text {exp }}\right)=1$. As such, the probabilities being calculated are the relative probabilities between the different dynamical models, which does not necessarily include the possibility of systematic errors. These limitations are considered in detail by Tsang in [15], where the different types of systematic errors are listed and discussed. This limitation does not invalidate the approach presented here. However, it does mean that experimental studies need to be careful to calibrate their systems fully and to verify that systematic errors are either not present, or are included explicitly in one of the dynamical models.

To summarize, given an experimental measurement record consisting of discrete increments $\Delta I_{\exp , t}$, and a set of dynamical models $m_{k}$ describing the possible evolution of the underlying system, we proceed as follows. At $t=0$, set initial probabilities for all models, with the default assumption being that all models are equally likely. At each subsequent time step,

(1) calculate the increment $\Delta W_{t}^{\left(m_{k}\right)}$ for each model using Eq. (6) and $\Delta I_{\exp , t}$, and update the corresponding conditional states using the appropriate form of Eq. (1);

(2) calculate the probability update $p\left(\Delta I_{\exp , t} \mid m_{k}\right)$ for each model using Eq. (5);

(3) update all probabilities, using Eq. (4);

(4) normalize to find relative probabilities; and

(5) repeat using next measurement increment, $\Delta I_{\exp , t+\Delta t}$.

Once the updates have been included from all measurements in the record, the decision processing given by Eq. (3) can be applied. One benefit of this procedure is that it is clearly iterative, and can therefore be used as an online process, with probabilities being updated as each measurement is taken; or, if required, as a postprocessing step after experimental data collection.

Quality of the decision. We have now introduced dynamical models and selection rules. In particular, we have discussed how to select the best model $m_{j} \in M$ given a measured signal $I_{\text {exp. }}$. However, the model selected might not be overall the best model to describe the experiment, e.g., taking a longer time trace $I_{\exp }$, or repeating the experiment several times, one might find out that the best model to describe the system is actually a different one. To estimate the probabilities of making a correct or a false selection, one can proceed using the following procedure.

Suppose the system evolves according to the model $m_{s}$. In the absence of an experimental record, one can generate a time trace $I_{\text {sim }}^{\left(m_{s}\right)}$ numerically, solving Eqs. (1) and (2), and using a Gaussian random number generator for the Wiener increments $d W$. After the time trace is generated, one uses the simulated increments to calculate the conditional state evolution for each of the models and generating relative probabilities given the simulated record, $P\left(m_{k} \mid I_{\text {sim }}^{\left(m_{s}\right)}\right)$. The most probable model is then selected using the Neyman-Pearson rule given in Eq. (3), given the simulated time trace $I_{\text {sim }}^{\left(m_{s}\right)}$. One repeats this procedure $N^{(s)}$ times to estimate the probabilities of false and correct identification:

$$
P\left(m_{k} \mid m_{s}\right) \approx \frac{N_{k}^{(s)}}{N^{(s)}}
$$

where $N_{k}^{(s)}$ denotes the number of times the model $m_{k}$ was selected, when the time trace $I_{\text {sim }}^{\left(m_{s}\right)}$ was generated using model $m_{s}$. In the limit $N^{(s)} \rightarrow \infty$, we obtain the probability $P\left(m_{k} \mid m_{s}\right)$ of selecting model $m_{k}$, when the time trace $I_{\text {sim }}^{\left(m_{s}\right)}$ has been generated using model $m_{s}$.

The probabilities $P\left(m_{k} \mid m_{s}\right)$ form the elements of the socalled confusion matrix $\left(M_{c}\right)_{s k}$ [27]. For example, in the case where we are considering only two models, e.g., a classical one and a quantum one, denoted by $C$ and $Q$ respectively, we can arrange the probabilities of correct and false identification in the following $2 \times 2$ matrix:

$$
M_{c}=\left(\begin{array}{ll}
p(C \mid C) & p(C \mid Q) \\
p(Q \mid C) & p(Q \mid Q)
\end{array}\right),
$$

where $p(C \mid Q)$ is the probability of a type-II error (false negative, assuming that the classical hypothesis $C$ is the default or null hypothesis) and $p(Q \mid C)$ is the probability of a type-I error (false positive). More generally, one can generate a receiver-operator characteristic curve [27] by varying the threshold value $\mu$.

Application to optomechanics. Levitated optomechanical systems are a topical area of research. They have been used for ultrasensitive force measurements [28], fundamental tests of gravity [29], as well as testing the limits of quantum mechanics [30-32]. Here we propose a type of experiment to detect nonclassical features in such systems using dynamical model selection.

For the purposes of this Rapid Communication, we assume that the motion of a levitated nanoparticle is decoupled along the three motional axes and discuss only one-dimensional motion $[21,33,34]$. Specifically, we suppose that the potential is nonlinear and it forms a Duffing oscillator [35-37]. The Hamiltonian is given by

$$
\hat{H}=\frac{1}{2} \hat{p}^{2}-\frac{1}{2} \omega^{2} \hat{q}^{2}+\frac{1}{4} \beta \hat{q}^{4}+g \cos (t) \hat{q},
$$

where we have taken $\hbar=1$, the mass is scaled so that $m=$ $1, \hat{p}$ is the momentum operator, $\omega$ is the angular frequency of a linear oscillator, $\beta$ is the nonlinear parameter, and $g$ is the magnitude of an external periodic drive. This system has been widely studied in relation to chaotic dynamics in open quantum systems and the quantum-classical transition [35-41]. The full quantum $(Q)$, as well as the corresponding classical $(C)$ model, are of the form given in Eqs. (1) and (2), with additional dissipator terms to describe the interactions with gas particles, acting as a thermal environment (see Appendix $\mathrm{B}$ for more details).

For the case considered here, one would like to find the conditions where one can best discriminate between the two dynamical models, and thus plan the experimental implementation accordingly. A number of different conditions were examined, for single-well (linear and nonlinear) and doublewell potentials. The optimum condition was found to be a double-well potential with $\omega=1, \beta=0.5$, and $g=0$ (see Appendix $\mathrm{C}$ for more details).

In general, the probabilities for correctly identifying a quantum system, $P(Q \mid Q)$, are slightly higher than for the classical system, $P(C \mid C)$. At low temperatures $k_{B} T<\Delta E_{01}$ the distinguishability is excellent, approaching $100 \%$ even for measurement efficiencies $\eta \simeq 0.2$, where $\Delta E_{01}$ is the energy 

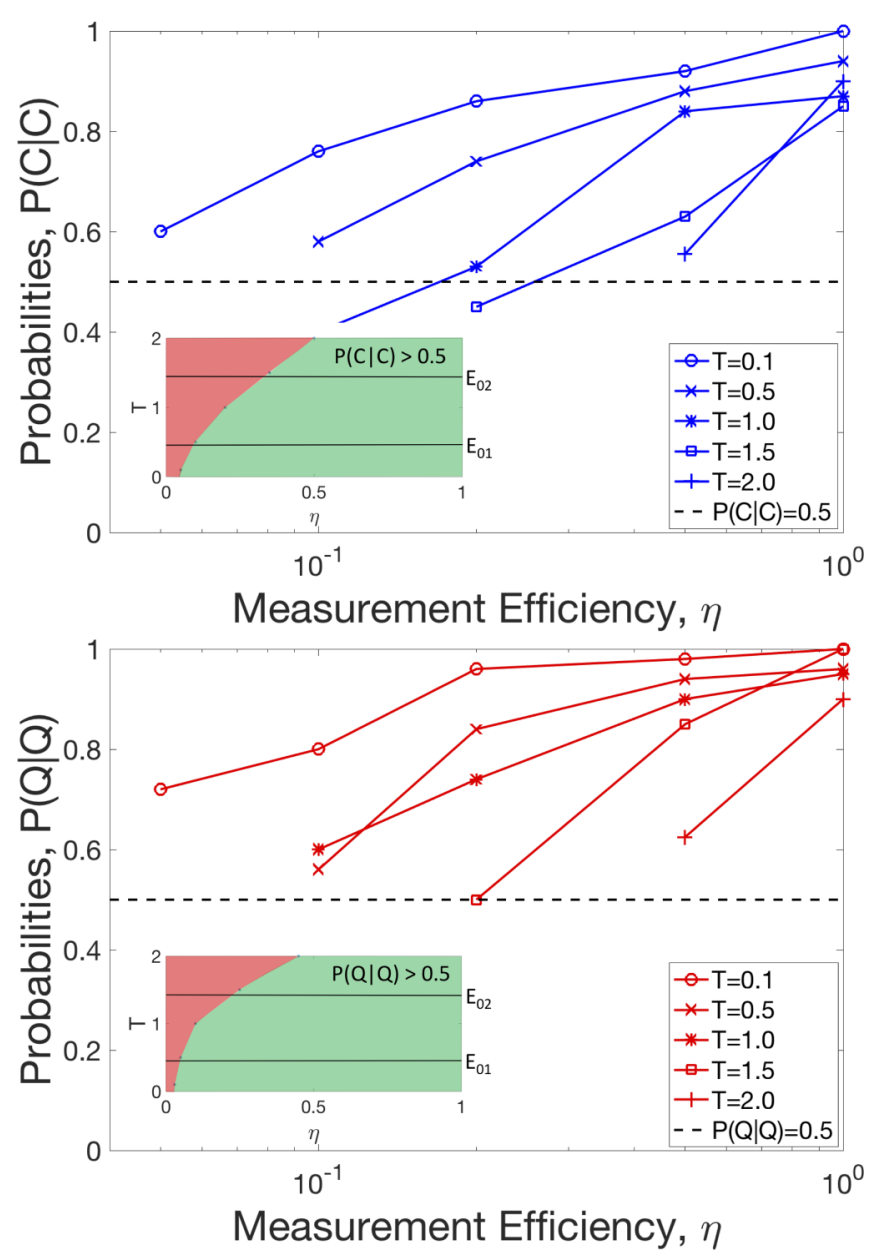

FIG. 1. Numerically calculated probabilities for the identification of the correct dynamical evolution of an optomechanical example with trapped nanoparticles in a double-well potential: $Q$ is calculated using a quantum stochastic master equation, and $C$ is calculated using the equivalent classical stochastic differential equation. Probabilities $p(C \mid C)$ and $p(Q \mid Q)$ are shown, for different temperatures $T$ as a function of the measurement efficiency $\eta$. Insets show approximate regions where the models are distinguishable [green (light-gray) shaded regions], as functions of temperature and measurement efficiency.

separation between the ground state and the first excited state. This contrasts with a linear trap, where the probability of correctly distinguishing dynamical models was found to be limited to around $80 \%$, even for very low temperatures and ideal measurements $\eta=1.0$. Here, with two wells, both dynamical models show good distinguishability between quantum and classical behavior for temperatures $T \simeq 0.5\left(k_{B} T \simeq \Delta E_{01}\right)$ and measurement efficiencies $\eta>0.2$, with some ability to distinguish between the two models for temperatures where the thermal energy is well above the first energy level separation and around the second transition, $k_{B} T \simeq 1.5 \hbar \omega \sim 4 \Delta E_{01}$, as long as $\eta>0.5$ (see Fig. 1).

Typical trapping frequencies in experiments are around $100 \mathrm{kHz}$ and masses of the nanoparticles are a few $\times 10^{-19} \mathrm{~kg}$ $[33,34]$. In this case, $\hbar \omega$ corresponds to a temperature of $0.77 \mu \mathrm{K}$, and $T=1.5 \simeq 1.16 \mu \mathrm{K}$, with the two wells separated by $0.2 \mathrm{~nm}$, smaller than the radius of the sphere. However, double-well optical traps can be generated using fabricated structures within a few nanometers spacing between the two wells, below the diffraction limit [42,43]. Similarly, a few nanometers of spacing in ion trapping using optical lattices is demonstrated in [44]. Alternatively, a double well can be generated by focusing two laser beams of different wavelengths [45]. A dielectric particle will thus evolve in an effective potential of these two partially overlapping potentials. As highlighted in $[42,46]$, trapped particles can have resolutions well below $\sim 1 \mathrm{pm}[33,34]$. Therefore, such double-well traps are realizable within the current experiments. Experiments with levitated nanoparticles have reported temperatures around $450 \mu \mathrm{K}$ [47], well above the regime required, but experimental techniques are improving rapidly and temperatures equivalent to $\bar{n} \sim 10-20$ are anticipated in the near future. Measurement efficiencies are more difficult to estimate from previous work since the values are not critical to the results presented and are not normally provided. However, for other systems, such as superconducting circuits [48-51], it is known that measurement efficiencies of at least $\eta \sim 0.4$ are achievable [49].

Conclusions. This Rapid Communication has discussed a general method to distinguish between dynamical models for quantum and classical systems. It provides an alternative to standard statistical tests based on state reconstruction. We have rephrased the problem of model selection in a form suitable to apply the well-known Neyman-Pearson decision rule, and quantified the reliability of the selection using the confusion matrix. Particularly noteworthy is the simplicity and generality of the proposed method: dynamical model selection is based on generic time-trace data and it could be used to select between a wide variety of dynamical models.

To illustrate the method of dynamical model selection, we have considered its application to levitated optomechanical systems, where nonclassical features are yet to be experimentally demonstrated. We have proposed and optimized an experiment, where the nanoparticle is optically trapped in a double-well potential. Using dynamical model selection we have provided limits for two key experimental parameters (temperature and measurement efficiency) for quantum behavior to be detected reliably. The successful experimental implementation, were it to confirm nonclassical features, would improve on the most massive particle shown to exhibit quantum interference by several orders of magnitude [52], and would thus be of great importance to fundamental physics.

Acknowledgments. J.F.R. would like to thank H. M. Wiseman, P. Barker, and M. Flinders for useful and informative discussions. M.R., M.T., A.S., and H.U. acknowledge funding by the Leverhulme Trust (RPG-2016-046), the EU Horizon 2020 FET project TEQ (Grant No. 766900), and the Foundational Questions Institute (FQXi), and A.S. acknowledges support by the Engineering and Physical Sciences Research Council (EPSRC) under Centre for Doctoral Training Grant No. EP/L015382/1.

\section{APPENDIX A: GENERAL DIFFUSIVE MODELS}

In this Appendix we consider nonrelativistic, Markovian, diffusive models [23,24]. We start by discussing general classical models. Specifically, the conditional probability density $P_{c}$ evolves according to the Kushner-Stratonovich 
equation [24]:

$$
\begin{aligned}
d P_{c}= & -\sum_{k}^{r=1} \frac{\partial}{\partial q_{k}}\left(a_{k} P_{c}\right) d t \\
& +\frac{1}{2} \sum_{k}^{r=1} \sum_{k^{\prime}=1}^{r} \frac{\partial}{\partial q_{k} \partial q_{k^{\prime}}}\left(D_{k k^{\prime}} P_{c}\right) d t \\
& +\sum_{k}^{r^{\prime}} \sum_{k^{\prime}=1}^{r^{\prime}}[c-\mathbb{E}[c]]_{k}\left(B B^{\top}\right)_{k k^{\prime}}[B d V+(c-\mathbb{E}[c]) d t]_{k^{\prime}},
\end{aligned}
$$

where $a_{k} \in \mathbb{R}, B_{k k^{\prime}} \in \mathbb{R}, c_{k} \in \mathbb{R}, D_{k k^{\prime}} \in \mathbb{R}$, and $V$ is a vector of mutually independent $\mathbb{R}$-valued Wiener processes. The first line corresponds to the Hamiltonian evolution, the second line to the diffusion, i.e., in case the measurement perturbs the system, and the third line to the update in the knowledge about the system. In particular, the measured signal is given by (an $r^{\prime}$-dimensional vector)

$$
d I_{\exp }=c d t+B d V .
$$

We next describe general quantum models. Specifically, the conditional statistical operator $\hat{\rho}_{c}$ evolves according to the Belavkin equation [22-24]:

$$
d \hat{\rho}_{c}=-i\left[\hat{H}, \rho_{c}\right] d t+\mathcal{D}[\hat{c}] \hat{\rho}_{c} d t+\mathcal{H}\left[d U^{\dagger} \hat{c}\right] \hat{\rho}_{c},
$$

where $\hat{c}$ is an $r^{\prime}$-dimensional vector of operators. $U$ is an $r^{\prime}$ dimensional vector of correlated $\mathbb{C}$-valued Wiener processes satisfying

$$
\begin{aligned}
d U d U^{\dagger} & =\eta d t, \\
d U d U^{\top} & =\Xi d t,
\end{aligned}
$$

where $\eta$ is diagonal with $\eta_{k k} \in[0,1]$, and $\Xi$ is symmetric with $\mathbb{C}$-valued elements. Moreover, we have the constraint that

$$
\left[\begin{array}{cc}
\eta+\operatorname{Re}(\Xi) & \operatorname{Im}(\Xi) \\
\operatorname{Im}(\Xi) & \eta+\operatorname{Re}(\Xi)
\end{array}\right]
$$

is postive semidefinite. Note that the first, second, and third terms on the right-hand side of Eq. (A3) correspond to the first, second, and third lines of the right-hand side of Eq. (A1), respectively. The measurement signal is given by (an $r^{\prime}$-dimensional vector with $\mathbb{C}$-valued elements)

$$
d I_{\text {exp }}=\operatorname{Tr}\left[\left(\hat{c}^{\top} \eta+\hat{c}^{\dagger} \Xi\right) \hat{\rho}_{c}\right] d t+d U^{\top} .
$$

\section{APPENDIX B: OPTOMECHANICAL SYSTEM MODELS}

For our purposes, the important factors are (i) a levitated nanoparticle is physically large (with a radius several hundred to a few thousand times that of an atom); (ii) a nanoparticle has a high mass (six to eight orders of magnitude larger than an atom); (iii) the trap can be arranged to separate degrees of freedom in terms of frequency, thereby simplifying the system to one translational degree of freedom; and (iv) the particle is weakly coupled to a thermal environment and to a laser field that can be used to provide a continuous measurement of position. We will take parameters based on optomechanical spheres described in $[33,34]$, made from silica with radii $\simeq 25-100 \mathrm{~nm}$ and masses $m \simeq 10^{-19}-10^{-18} \mathrm{~kg}$. These are good candidates for study because they previously have been used in experiments to generate thermal squeezed states [33], measurements have been used to reconstruct (classical) Wigner functions [34], and they can realize the multiple-well potentials [45], which we find maximizes the discrimination between classical and quantum models.

A continuous quantum measurement process is usually modeled with a stochastic master equation (SME) [22-24], which can be written as

$$
\begin{aligned}
d \rho_{c}= & -i\left[\hat{H}, \rho_{c}\right] d t \\
& +\sum_{r=1}^{m^{\prime}}\left\{\hat{L}_{r} \rho_{c} \hat{L}_{r}^{\dagger}-\frac{1}{2}\left(\hat{L}_{r}^{\dagger} \hat{L}_{r} \rho_{c}+\rho_{c} \hat{L}_{r}^{\dagger} \hat{L}_{r}\right)\right\} d t \\
& +\sum_{r=1}^{m^{\prime}} \sqrt{\eta_{r}}\left(\hat{L}_{r} \rho_{c}+\rho_{c} \hat{L}_{r}^{\dagger}-\operatorname{Tr}\left(\hat{L}_{r} \rho_{c}+\rho_{c} \hat{L}_{r}^{\dagger}\right)\right) d W_{r},
\end{aligned}
$$

where $\rho_{c}$ is the density matrix for the state of the system conditioned on the measurement record - the state (possibly mixed) which represents the current knowledge of the quantum state, $\hat{H}$ is the Hamiltonian of the system, $d t$ is an infinitesimal time increment, and the operators $\hat{L}_{r}$ represent the effect of the environment and measurement. The measurement record for each of the operators $\hat{L}_{r}$ during a time step $t \rightarrow t+$ $d t$ is given by $y(t+d t)-y(t)=d y_{r}(t)=\sqrt{\eta_{r}} \operatorname{Tr}\left(\hat{L}_{r} \rho_{c}+\right.$ $\left.\rho_{c} \hat{L}_{r}^{\dagger}\right) d t+d W_{r}$, where the recorded time trace in this interval is $d I_{\exp }(t)=d y(t) / \sqrt{2 k} \cdot \eta_{r}$ is the measurement efficiency; the ratio of the signal power due to the measurement relative to the power of other extraneous sources of noise, where $\eta_{r}=1$ is an ideal measurement and $\eta_{r}=0$ is an unprobed environmental degree of freedom. Moreover, we will assume that $d W_{r}$ are independent real Wiener processes, i.e., $d W_{r} d W_{r^{\prime}}=\delta_{r r^{\prime}} d t$. Physically, this SME represents a situation where the measurement environment decoheres sufficiently rapidly that no correlations build up between the state of the quantum system of interest and the environmental degrees of freedom (Markov approximation).

For the case considered here, the SME is given by (B1) with three environmental operators $\left(m^{\prime}=3\right)$ : one measurement of the position $(q)$ of the nanosphere within the trap, $\hat{L}_{1}=\sqrt{2 k} \hat{q}$, and two operators representing an unprobed thermal environment $\hat{L}_{2}=\sqrt{(\bar{n}+1) \Gamma} \hat{a}^{\dagger}$ and $\hat{L}_{3}=\sqrt{\bar{n} \Gamma} \hat{a}$ [53], where $\hat{a}^{\dagger}$ and $\hat{a}$ are the usual harmonic oscillator raising and lowering operators, $\Gamma$ is a decay rate $(\Gamma \ll \omega), \bar{n}=\left[\exp \left(\hbar \omega / k_{B} T\right)-1\right]^{-1}$ is the average thermal occupation number of a linear oscillator at temperature $T$, and $k$ is the measurement strength for the continuous measurement interaction. The measurement efficiencies are $\eta_{1}=\eta$ and $\eta_{2,3}=0$ (unprobed). The measurement record for $\hat{L}_{1}$ is $d y(t)=\sqrt{8 \eta k} \operatorname{Tr}\left[\rho_{c}(t) \hat{q}\right] d t+d W$.

For the equivalent classical system, we take a stochastic differential equation (SDE) for the position $q$ and the momentum $p$ of a classical particle,

$$
\begin{aligned}
d q= & p d t \\
d p= & -\mu q^{3} d t+\omega^{2} q d t-\Gamma p d t+g \cos (t) \\
& +\sqrt{2 k} d Y+\sqrt{2 \Gamma k_{B} T} d U,
\end{aligned}
$$


where the measurement record is $d y_{c}(t)=\sqrt{8 \eta k} q d t+d W$ and we have again set $\hbar=1$. Like $d W, d Y$ and $d U$ are also real Weiner increments, $d Y^{2}=d U^{2}=d t$, but they are uncorrelated so that $d W d U=d W d Y=d Y d U=0$, and there is no backaction from the measurement on the state of the system in a classical measurement.

\section{APPENDIX C: OPTOMECHANICAL SYSTEM SIMULATION}

The distinguishibility of the two models was found to be best in the double-well configuration. Specifically, when the two wells were well separated in position and the barrier between the two wells was high enough for the classical particle to remain in one well for a reasonable period of time, before the environmental noise kicked it into the other well. In addition, the barrier also had to be low enough to prevent the quantum state localizing in one or other of the wells. In practice, these conditions correspond to a symmetric double-well potential where the quantum ground state lies below the barrier height but the first excited state is above the barrier. The classical system is always localized, in the sense that it is a point particle, but the probability density function (pdf) represented by the particles needs to be largely localized to one of the wells by the measurements. By contrast, a quantum state can only be localized to one of the wells if two of the low-lying energy levels are below the barrier. If the barrier is sufficiently high, the lowest two energy states are formed from the symmetric and antisymmetric superposition of localized well states, and a localized well state can be generated by combining these two energy levels [54]. If the first excited state lies above the barrier, then a superposition of this with the ground state will not be localized in one well. For the Duffing Hamiltonian (9), these conditions are met if we take $\omega=1, \beta=0.5$, and $g=0$, where we have set $\Gamma=0.05, k=0.025$, and $N=500$.

The quantum model uses Rouchon's integration method [55,56] with noncommutative noise sources and a moving basis [35-37] with 60-100 linear oscillator states. The models are integrated over 100 cycles of the linear oscillator with 500-2000 time steps per oscillator cycle, and the probabilities are calculated by averaging over 100 runs of each model. The barrier height in this example is $\Delta E_{b}=0.5 \hbar \omega$, the two wells are separated in position by $\Delta q=3 \sqrt{\hbar / m \omega}$, the lowest two energy levels are separated by $\Delta E_{01}=0.396 \hbar \omega$, and the next excited states are separated by $\Delta E_{12}=0.941 \hbar \omega$ and $\Delta E_{23}=1.061 \hbar \omega$.

The classical model requires the evolution of the pdf to be calculated, which is computationally expensive. We use an alternative approach here to solve the approximate problem using a sequential Monte Carlo method [26,57,58] known as a particle filter. The particle filter uses the fact that the evolution of the pdf can be approximated by the evolution of a finite number of candidate solutions or "particles," each of which has a weight associated with it, where the weight evolves in such a way that a quantity averaged over all weighted particles approximates the expectation value for the quantity over the pdf. In this case, we take $N$ particles, initialized with equal weight $w_{0}^{(i)}=1 / N$. Each particle has a position $q^{(i)}$ and a momentum $p^{(i)}$, initially selected from the same thermal distribution as that given by the thermal state for the quantum model. The particles then evolve according to the SDE (B2) with independent noise sources. The weights are updated using

$$
\tilde{w}_{t}^{(i)}=\frac{\exp \left(-\frac{\left(\Delta \tilde{y}_{t}-\sqrt{8 \eta k} q^{(i)} \Delta t\right)^{2}}{2 \Delta t}\right)}{\sqrt{2 \pi \Delta t}} w_{t-\Delta t}^{(i)},
$$

where the $\tilde{w}^{(i)}$ 's are unnormalized weights after updating, and the probability for the classical model is approximated by $p\left(\Delta \tilde{y}_{t} \mid \mathrm{C}, \Delta \tilde{y}_{0: t^{\prime}-\Delta t}\right) \propto \sum_{i=1}^{N} \tilde{w}_{t}^{(i)}$. As the system evolves, the values of some of the weights fall to near zero. The particles and the candidate solutions that they represent are then resampled using the current weight distribution as described in [16]. This evolution with periodic resampling allows the particle filter to be efficient while still retaining a diverse selection of candidate solutions. This makes the particle filter an ideal method for the estimation of a nonlinear dynamical process and it is the reason for considering it in a model selection context. In addition, the particle filter and other sequential Monte Carlo methods can be augmented to include the simultaneous estimation of system parameters [59] and they can be applied to quantum systems described by SMEs with uncertain parameters [60].

It should be noted that the ability to distinguish the models is dependent on the total time over which the measurement record is collected and the models integrated. Extending the integration time will improve the results, but the trap potential and the measurement interaction would need to be stable over the integration time, providing a trade-off between distinguishability and difficulties in collecting the measurement data.
[1] O. Nairz, M. Arndt, and A. Zeilinger, Am. J. Phys. 71, 319 (2003).

[2] S. Gerlich, S. Eibenberger, M. Tomandl, S. Nimmrichter, K. Hornberger, P. J. Fagan, J. Tuxen, M. Mayor, and M. Arndt, Nat. Commun. 2, 263 (2011).

[3] D. T. Smithey, M. Beck, M. G. Raymer, and A. Faridani, Phys. Rev. Lett. 70, 1244 (1993).

[4] A. I. Lvovsky, H. Hansen, T. Aichele, O. Benson, J. Mlynek, and S. Schiller, Phys. Rev. Lett. 87, 050402 (2001).

[5] C. F. Roos, G. P. T. Lancaster, M. Riebe, H. Haffner, W. Hansel, S. Gulde, C. Becher, J. Eschner, F.
Schmidt-Kaler, and R. Blatt, Phys. Rev. Lett. 92, 220402 (2004)

[6] K. J. Resch, P. Walther, and A. Zeilinger, Phys. Rev. Lett. 94, 070402 (2005).

[7] Y. Nakamura, C. D. Chen, and J. S. Tsai, Phys. Rev. Lett. 79, 2328 (1997).

[8] J. R. Friedman, V. Patel, W. Chen, S. K. Tolpygo, and J. E. Lukens, Nature (London) 406, 43 (2000).

[9] C. H. van der Wal, A. C. J. ter Haar, F. K. Wilhem, R. N. Schouten, C. J. P. M. Harmans, T. P. Orlando, S. Lloyd, and J. E. Mooij, Science 290, 773 (2000). 
[10] J. M. Martinis, S. Nam, J. Aumentado, and C. Urbina, Phys. Rev. Lett. 89, 117901 (2002).

[11] M. Barbieri, F. De Martini, G. Di Nepi, Paolo Mataloni, G. M. D’Ariano, and C. Macchiavello, Phys. Rev. Lett. 91, 227901 (2003).

[12] M. Bourennane, M. Eibl, C. Kurtsiefer, S. Gaertner, H. Weinfurter, O. Gühne, P. Hyllus, D. Bruß, M. Lewenstein, and A. Sanpera, Phys. Rev. Lett. 92, 087902 (2004).

[13] C.-Y. Lu, X.-Q. Zhou, O. Gühne, W.-B. Gao, J. Zhang, Z.-S. Yuan, A. Goebel, T. Yang, and J.-W. Pan, Nat. Phys. 3, 91 (2007).

[14] M. Tsang, Phys. Rev. Lett. 108, 170502 (2012).

[15] M. Tsang, Quant. Meas. Quant. Metrol. 1, 84 (2013).

[16] N. Gordon, S. Maskell, and T. Kirubarajan, Efficient Particle Filters for Joint Tracking and Classification, in Proceedings of the SPIE: Signal and Data Processing of Small Targets (SPIE, 2002), pp. 439-449.

[17] A. Ashkin, Phys. Rev. Lett. 24, 156 (1970).

[18] S. Mancini, D. Vitali, and P. Tombesi, Phys. Rev. Lett. 80, 688 (1998).

[19] M. Gangl and H. Ritsch, Phys. Rev. A 61, 011402 (1999).

[20] V. Vuletic and S. Chu, Phys. Rev. Lett. 84, 3787 (2000).

[21] M. Toroš, M. Rashid, and H. Ulbricht, arXiv:1804.01150.

[22] V. P. Belavkin, Rep. Math. Phys. 43, 353 (1999).

[23] H. M. Wiseman and G. J. Milburn, Quantum Measurement and Control' (Cambridge University Press, Cambridge, 2010).

[24] K. Jacobs Quantum Measurement Theory and Its Applications (Cambridge University Press, Cambridge, 2014).

[25] H. M. Wiseman and G. J. Milburn, Phys. Rev. A 47, 1652 (1993).

[26] M. Arulampalam, S. Maskell, N. Gordon, and T. Clapp, IEEE Trans. Signal Process. 50, 241 (2002).

[27] D. J. Hand and R. J. Till, Machine Learning 45, 171 (2001).

[28] A. A. Geraci, S. B. Papp, and J. Kitching, Phys. Rev. Lett. 105, 101101 (2010).

[29] A. Arvanitaki and A. A. Geraci, Phys. Rev. Lett. 110, 071105 (2013).

[30] A. Bassi and G. C. Ghirardi, Phys. Rep. 379, 257 (2003).

[31] A. Bassi, K. Lochan, S. Satin, T. P. Singh, and H. Ulbricht, Rev. Mod. Phys. 85, 471 (2013).

[32] S. Bera, B. Motwani, T. P. Singh, and H. Ulbricht, Sci. Rep. 5, 7664 (2015).

[33] M. Rashid, T. Tufarelli, J. Bateman, J. Vovrosh, D. Hempston, M. S. Kim, and H. Ulbricht, Phys. Rev. Lett. 117, 273601 (2016).

[34] M. Rashid, M. Toroš, and H. Ulbricht, Quant. Meas. Quant. Metrol. 4, 17 (2017).

[35] R. Schack, T. A. Brun, and I. C. Percival, J. Phys. A: Math. Gen. 28, 5401 (1995).

[36] T. A. Brun, I. C. Percival, and R. Schack, J. Phys. A: Math. Gen. 29, 2077 (1996).

[37] J. F. Ralph, K. Jacobs, and M. J. Everitt, Phys. Rev. A 95, 012135 (2017).
[38] T. Bhattacharya, S. Habib, and K. Jacobs, Phys. Rev. A 67, 042103 (2003).

[39] M. J. Everitt, T. D. Clark, P. B. Stiffell, J. F. Ralph, A. R. Bulsara, and C. J. Harland, New J. Phys. 7, 64 (2005).

[40] J. K. Eastman, J. J. Hope, and A. R. R. Carvalho, Sci. Rep. 7, 44684 (2017).

[41] B. Pokharel, P. Duggins, M. Misplon, W. Lynn, K. Hallman, D. Anderson, A. Kapulkin, and A. K. Pattanayak, Sci. Rep. 8, 2108 (2018).

[42] Y. Tanaka, S. Kaneda, and K. Sasaki, Nano Lett. 13, 2146 (2013).

[43] A. Kotnala, D. DePaoli, and R. Gordon, Lab Chip 13, 4142 (2013).

[44] Y. Wang, S. Subhankar, P. Bienias, M. Lacki, T.-C. Tsui, M. A. Baranov, A. V. Gorshkov, P. Zoller, J. V. Porto, and S. L. Rolston, Phys. Rev. Lett. 120, 083601 (2018).

[45] L. Rondin, J. Gieseler, F. Ricci, R. Quidant, and C. Dellago. L. Novotny, Nat. Nanotechnol. 12, 1130 (2017).

[46] J. Vovrosh, M. Rashid, D. Hempston, J. Bateman, M. Paternostro, and H. Ulbricht, J. Opt. Soc. Am. B 34, 1421 (2017).

[47] V. Jain, J. Gieseler, C. Moritz, C. Dellago, R. Quidant, and L. Novotny, Phys. Rev. Lett. 116, 243601 (2016).

[48] K. W. Murch, S. J. Weber, C. Macklin, and I. Siddiqi, Nature (London) 502, 211 (2013).

[49] S. J. Weber, A. Chantasri, J. Dressel, A. N. Jordan, K. W. Murch, and I. Siddiqi, Nature (London) 511, 570 (2014).

[50] P. Six, P. Campagne-Ibarcq, L. Bretheau, B. Huard, and P. Rouchon, Proceedings of the 54th IEEE Conference on Decision and Control Conference (CDC) (IEEE, Osaka, 2015).

[51] P. Campagne-Ibarcq, P. Six, L. Bretheau, A. Sarlette, M. Mirrahimi, P. Rouchon, and B. Huard, Phys. Rev. X 6, 011002 (2016).

[52] S. Eibenberger, S. Gerlich, M. Arndt, M. Mayor, and J. Tüxen, Phys. Chem. Chem. Phys. 15, 14696 (2013).

[53] T. P. Spiller, B. M. Garraway, and I. C. Percival, Phys. Lett. A 179, 63 (1993).

[54] M. J. Everitt, T. D. Clark, P. B. Stiffell, A. Vourdas, J. F. Ralph, R. J. Prance, and H. Prance, Phys. Rev. A 69, 043804 (2004).

[55] H. Amini, M. Mirrahimi, and P. Rouchon, Proceedings of the 50th IEEE Conference on Decision and Control (IEEE, Orlando, 2011), pp. 6242-6247.

[56] P. Rouchon and J. F. Ralph, Phys. Rev. A 91, 012118 (2015).

[57] N. Gordon, D. Salmond, and A. F. Smith, IEE Proc., Part F 140, 107 (1993).

[58] A. Doucet, N. De Freitas, and N. Gordon, Eds., Sequential Monte Carlo Methods in Practice (Springer, New York, 2001).

[59] P. L. Green and S. Maskell, Mech. Syst. Signal Process. 93, 379 (2017).

[60] J. F. Ralph, S. Maskell, and K. Jacobs, Phys. Rev. A 96, 052306 (2017). 